UPR-0983T

FT 2002-03

MADPH-02-1266

\title{
The $Z-Z^{\prime}$ Mass Hierarchy in a Supersymmetric Model with a Secluded $U(1)^{\prime}$-Breaking Sector
}

\author{
Jens Erler ${ }^{1,2}$, Paul Langacker ${ }^{1,3}$ and Tianjun $\operatorname{Li}^{1}$ \\ ${ }^{1}$ Department of Physics and Astronomy \\ University of Pennsylvania, Philadelphia, PA 19104-6396 \\ ${ }^{2}$ Universidad Nacional Autónoma de México \\ Instituto de Física, 01000 México D.F. \\ ${ }^{3}$ Department of Physics, University of Wisconsin, Madison WI 53706
}

\begin{abstract}
We consider the $Z^{\prime} / Z$ mass hierarchy in a supersymmetric model in which the $U(1)^{\prime}$ is broken in a secluded sector coupled to the ordinary sector only by gauge and possibly soft terms. A large mass hierarchy can be achieved while maintaining the normal sparticle spectra if there is a direction in which the tree level potential becomes flat when a particular Yukawa coupling vanishes. We describe the conditions needed for the desired breaking pattern, to avoid unwanted global symmetries, and for an acceptable effective $\mu$ parameter. The electroweak breaking is dominated by $A$ terms rather than scalar masses, leading to $\tan \beta \simeq 1$. The spectrum of the symmetry breaking sector is displayed. There is significant mixing between the MSSM particles and new standard model singlets, for both the Higgs scalars and the neutralinos. A larger Yukawa coupling for the effective $\mu$ parameter is allowed than in the NMSSM because of the $U(1)^{\prime}$ contribution to the running from a high scale. The upper bound on the tree-level mass of the lightest CP even Higgs doublet mass is about $c \times 174 \mathrm{GeV}$, where $c$ is of order unity, but the actual mass eigenvalues are generally smaller because of singlet mixing.
\end{abstract}

October 25, 2018 


\section{Introduction}

The possibility of an extra $U(1)^{\prime}$ gauge symmetry is well-motivated in superstring constructions [1] and grand unified theories [2], and also in models of dynamical symmetry breaking [3]. In supersymmetric models, an extra $U(1)^{\prime}$ can provide an elegant solution to the $\mu$ problem [4, 5], with an effective $\mu$ parameter generated by the vacuum expectation value (VEV) of the Standard Model (SM) singlet field $S$ which breaks the $U(1)^{\prime}$ symmetry. This is somewhat similar to the effective $\mu$ parameter in the Next to Minimal Supersymmetric Standard Model (NMSSM) [6]. However, with a $U(1)^{\prime}$ the extra discrete symmetries and their associated cosmological problems typically associated with the NMSSM are absent. A closely related feature is that the MSSM upper bound of $M_{Z}$ on the tree-level mass of the corresponding lightest MSSM Higgs scalar is relaxed, both in models with a $U(1)^{\prime}$ and in the NMSSM, because of the Yukawa term $h S H_{1} H_{2}$ in the superpotential and the $U(1)^{\prime} D$-term [7]. More generally, for specific $U(1)^{\prime}$ charge assignments for the ordinary and exotic fields one can simultaneously ensure the absence of anomalies; that all fields of the $\mathrm{TeV}$ scale effective theory are chiral, avoiding a generalized $\mu$ problem; and the absence of dimension-4 proton decay operators [8].

In superstring-motivated models it is often the case that electroweak and $U(1)^{\prime}$ breaking are both driven by soft supersymmetry breaking parameters, so one typically expects the $Z^{\prime}$ mass or masses to be of the same order as the electroweak scaleף, i.e., less than a TeV or so [1], so that such particles, if they exist, should be easily observed and their couplings studied at future colliders or at the Tevatron [10]. The typical expectation is that the $Z^{\prime}$ mass should be comparable to $M_{W}$ and $M_{Z}$. However, there are stringent limits from direct searches during Run I at the Tevatron [11] and from indirect precision tests at the $Z$-pole, at LEP 2, and from weak neutral current experiments [12]. The constraints depend on the particular $Z^{\prime}$ couplings, but in typical models one requires $M_{Z^{\prime}}>(500-800) \mathrm{GeV}$ and the $Z-Z^{\prime}$ mixing angle $\alpha_{Z-Z^{\prime}}$ to be smaller than a few $\times 10^{-3}$. (There are actually hints of deviations from the standard model in the $\mathrm{NuTeV}$ experiment [13] and in atomic parity violation [14, which could possibly be early signs of a $Z^{\prime}[15]$.) The non-observation to date of a $Z^{\prime}$ reduces the attractiveness of such scenarios, but does not exclude them. It has been shown in a number of examples [16] that there are small but not overly tuned corners of parameter space which can yield acceptable $Z^{\prime}$ parameters. The most common situation is that the soft-supersymmetry breaking parameters with dimensions of mass, and therefore the VEV of the field $S$ which breaks the $U(1)^{\prime}$, are large compared to the electroweak scale, e.g., of $O(\mathrm{TeV})$. The values of the Higgs doublet VEVs, and therefore $M_{W, Z}$, are relatively small by accidental cancellations. Since the SUSYbreaking scale is large in such scenarios, they typically lead to nonstandard sparticle spectra, with heavier squarks and sleptons than for most of the MSSM parameter space, but a richer spectra of Higgses, neutralinos, and usually charginos [17]. Another

\footnotetext{
${ }^{1}$ One way to avoid this conclusion is for the $U(1)^{\prime}$ breaking to occur along a direction which is $F$ and $D$ flat at the renormalizable tree level [9].
} 
possibility [16] is that the electroweak and $U(1)^{\prime}$ breaking are driven by $A$ terms that are relatively large compared to the typical soft scalar mass scale. This can lead to a small $\alpha_{Z-Z^{\prime}}$ and also a small $Z^{\prime}$ mass. The latter might be acceptable if the $Z^{\prime}$ has strongly suppressed couplings to leptons (perhaps after taking kinetic mixing [18] into account).

In this paper we consider another possibility, in which all of the dimensional SUSY-breaking parameters are at or below the electroweak scale, as is the VEV of the field which generates the effective $\mu$ term. Thus, the squark and slepton spectra can mimic those of the MSSM. The electroweak breaking is actually driven by electroweak scale $A$ terms, with the Higgs doublet and singlet masses smaller. A large $Z^{\prime}$ mass can be generated by the VEVs of additional SM singlet fields that are charged under the $U(1)^{\prime}$. If these fields are only weakly coupled to the SM fields, i.e., by $U(1)^{\prime}$ interactions and possibly soft SUSY-breaking terms, then the scale of VEVs in this sector is only weakly linked to the electroweak scale. In particular, we consider the situation in which there is an almost $F$ and $D$ flat direction involving these secluded fields, with the flatness lifted by a small Yukawa coupling. For a sufficiently small value for this Yukawa coupling, the $Z^{\prime}$ mass can be arbitrarily large. The class of models considered is related to the intermediate scale models considered in [9], except that in the latter case the flatness was lifted by higher dimensional operators or radiative corrections.

We choose the $U(1)^{\prime}$ charges so that off-diagonal soft supersymmetry breaking mass-square terms can avoid unwanted global symmetries, and show that there are only three such models up to charge conjugation. We describe two of these in detail, paying special attention to avoiding unphysical minima and runaway directions. Within our assumption of no special adjustment of parameters to achieve a moderate hierarchy in the ordinary sector, we find that the Yukawa coupling associated with the effective $\mu$ parameter must be relatively large, i.e., of $O(0.5-0.8)$. The upper end of this range would lead to a Landau pole in the NMSSM if one required the theory to be valid up to a large unification scale [19], but is acceptable in the $U(1)^{\prime}$ model due to the new contributions to the renormalization group equations. (It would be acceptable in either case if one did not require a canonical desert, as in models with large extra dimensions.) This scenario typically generates $\tan \beta \sim 1$, where $\tan \beta$ is the usual ratio of Higgs doublet VEVs; that the VEV of $S$ is comparable to that of the doublets; and that the upper bound on the lightest CP even Higgs doublet treelevel mass is of order $170 \mathrm{GeV}$. However, the actual mass eigenvalues are reduced by mixing with $S U(2)$ singlets. For these models, we display the spectra associated with the symmetry breaking, i.e., the gauge bosons, Higgses, neutralinos, and charginos. There is significant mixing between the standard model particles and SM singlets in the Higgs and neutralino sectors. We do not attempt to embed the models in a full theory or speculate on the small Yukawa couplings needed either for the large $Z^{\prime}$ mass or for fermions other than the $t$ quark. In the next section, we discuss the general features of this class of models. In Section 3 we calculate the spectra for typical $U(1)^{\prime}$ charges and parameter values. Our conclusions are given in Section 4 . Details of the minimization and Higgs mass-square matrices for one model are given in Appendix 
A, and the eigenvectors for the symmetry breaking sector are displayed for one model in Appendix B.

\section{The Chiral Supersymmetric $S U(3)_{C} \times S U(2) \times U(1) \times$ $U(1)^{\prime}$ Model}

We consider the supersymmetric $S U(3)_{C} \times S U(2) \times U(1) \times U(1)^{\prime}$ model with 2 Higgs doublets $\left(H_{1}\right.$ and $\left.H_{2}\right)$ and 4 Higgs singlets $\left(S, S_{1}, S_{2}\right.$, and $\left.S_{3}\right)$. The superpotential is 2

$$
W=h S H_{1} H_{2}+\lambda S_{1} S_{2} S_{3}
$$

where the Yukawa couplings $h$ and $\lambda$ are respectively associated with the effective $\mu$ term and with the runaway direction. The corresponding $F$-term scalar potential is

$$
\begin{aligned}
V_{F}= & h^{2}\left(\left|H_{1}\right|^{2}\left|H_{2}\right|^{2}+|S|^{2}\left|H_{1}\right|^{2}+|S|^{2}\left|H_{2}\right|^{2}\right) \\
& +\lambda^{2}\left(\left|S_{1}\right|^{2}\left|S_{2}\right|^{2}+\left|S_{2}\right|^{2}\left|S_{3}\right|^{2}+\left|S_{3}\right|^{2}\left|S_{1}\right|^{2}\right),
\end{aligned}
$$

The $D$-term scalar potential is

$$
\begin{aligned}
V_{D}= & \frac{G^{2}}{8}\left(\left|H_{2}\right|^{2}-\left|H_{1}\right|^{2}\right)^{2} \\
& +\frac{1}{2} g_{Z^{\prime}}^{2}\left(Q_{S}|S|^{2}+Q_{H_{1}}\left|H_{1}\right|^{2}+Q_{H_{2}}\left|H_{2}\right|^{2}+\sum_{i=1}^{3} Q_{S_{i}}\left|S_{i}\right|^{2}\right)^{2},
\end{aligned}
$$

where $G^{2}=g_{1}^{2}+g_{2}^{2} ; g_{1}, g_{2}$, and $g_{Z^{\prime}}$ are the coupling constants for $U(1), S U(2)$ and $U(1)^{\prime}$; and $Q_{\phi}$ is the $U(1)^{\prime}$ charge of the field $\phi$.

In addition, we introduce the supersymmetry breaking soft terms

$$
\begin{aligned}
V_{\text {soft }}^{(a)}= & m_{H_{1}}^{2}\left|H_{1}\right|^{2}+m_{H_{2}}^{2}\left|H_{2}\right|^{2}+m_{S}^{2}|S|^{2}+\sum_{i=1}^{3} m_{S_{i}}^{2}\left|S_{i}\right|^{2} \\
& -\left(A_{h} h S H_{1} H_{2}+A_{\lambda} \lambda S_{1} S_{2} S_{3}+\text { H.C. }\right)
\end{aligned}
$$

There are six neutral complex scalar fields and (in the general case) four phase symmetries of the scalar potential. Two of these are the $U(1)$ and $U(1)^{\prime}$ gauge symmetries, implying two unwanted global symmetries. These will generally be spontaneously broken, implying two massless Goldstone bosons. One of these has large

\footnotetext{
${ }^{2}$ One might consider a model with 3 singlets; for example, one can identify $S$ with $S_{1}$. The problem is that the $F$-term of $S$ is then $h H_{1} H_{2}+\lambda S_{2} S_{3}$. Depending on the soft parameters, there will either be a runaway direction for the scalar potential that is unbounded from below, an unphysical minimum with one of the Higgs doublet VEVs vanishing, or a minimum in which the VEVs of $H_{1}^{0}, H_{2}^{0}, S, S_{2}$ and $S_{3}$ are typically of the same order, preventing a $Z-Z^{\prime}$ mass hierarchy.
} 
$H_{1}^{0}$ and $H_{2}^{0}$ components and is clearly excluded by experiment. The second consists mainly of the $S_{i}$ fields, which couple to ordinary matter only by $U(1)^{\prime}$. These are most likely also excluded, though a detailed investigation is beyond the scope of this paper. We therefore consider special choices for the $U(1)^{\prime}$ charges which allow additional (off-diagonal) scalar mass-square terms which explicitly break the global symmetries.

For the models considered, one can take $A_{h}$ and $A_{\lambda}$ to be positive and the extra mass terms added to break the global symmetries to be negative by an appropriate redefinition of the scalar fields, without loss of generality. Then all of the VEVs can be taken to be real and positive at the minima. We define

$$
\left\langle H_{1}^{0}\right\rangle \equiv v_{1},\left\langle H_{2}^{0}\right\rangle \equiv v_{2}, \tan \beta=\frac{v_{2}}{v_{1}}
$$

and

$$
\langle S\rangle \equiv s,\left\langle S_{i}\right\rangle \equiv s_{i}
$$

Note that we have defined these VEVs without pulling out a factor of $1 / \sqrt{2}$, so the observed value of the electroweak scale is $\sqrt{v_{1}^{2}+v_{2}^{2}} \simeq 174 \mathrm{GeV}$. We also introduce

$$
\Delta \equiv Q_{S} s^{2}+Q_{H_{1}} v_{1}^{2}+Q_{H_{2}} v_{2}^{2}+\sum_{i=1}^{3} Q_{S_{i}} s_{i}^{2}
$$

The expressions for the chargino, neutralino, and $Z-Z^{\prime}$ mass matrices are independent of the forms of the supersymmetry breaking soft terms. The $Z-Z^{\prime}$ mass matrix is

$$
M_{Z-Z^{\prime}}=\left(\begin{array}{cc}
M_{Z}^{2} & M_{Z Z^{\prime}}^{2} \\
M_{Z Z^{\prime}}^{2} & M_{Z^{\prime}}^{2}
\end{array}\right)
$$

where

$$
\begin{gathered}
M_{Z}^{2}=\frac{G^{2}}{2}\left(v_{1}^{2}+v_{2}^{2}\right), M_{Z^{\prime}}^{2}=2 g_{Z^{\prime}}^{2}\left(Q_{S}^{2} s^{2}+Q_{H_{1}}^{2} v_{1}^{2}+Q_{H_{2}}^{2} v_{2}^{2}+\sum_{i=1}^{3} Q_{S_{i}}^{2} s_{i}^{2}\right), \\
M_{Z Z^{\prime}}^{2}=g_{Z^{\prime}} G\left(Q_{H_{1}} v_{1}^{2}-Q_{H_{2}} v_{2}^{2}\right) .
\end{gathered}
$$

The mass eigenvalues are

$$
M_{Z_{1}, Z_{2}}^{2}=\frac{1}{2}\left(M_{Z}^{2}+M_{Z^{\prime}}^{2} \mp \sqrt{\left(M_{Z}^{2}-M_{Z^{\prime}}^{2}\right)^{2}+4 M_{Z Z^{\prime}}^{4}}\right)
$$

and the $Z-Z^{\prime}$ mixing angle $\alpha_{Z-Z^{\prime}}$ is given by

$$
\alpha_{Z-Z^{\prime}}=\frac{1}{2} \arctan \left(\frac{2 M_{Z Z^{\prime}}^{2}}{M_{Z^{\prime}}^{2}-M_{Z}^{2}}\right),
$$


which is constrained to be less than a few times $10^{-3}$.

In the basis $\left\{\tilde{B}^{\prime}, \tilde{B}, \tilde{W}_{3}^{0}, \tilde{H}_{1}^{0}, \tilde{H}_{2}^{0}, \tilde{S}, \tilde{S}_{1}, \tilde{S}_{2}, \tilde{S}_{3}\right\}$, the neutralino mass matrix is

$$
M_{\tilde{\chi}^{0}}=\left(\begin{array}{cc}
M_{\tilde{\chi}^{0}}(6,6) & M_{\tilde{\chi}^{0}}(6,3) \\
M_{\tilde{\chi}^{0}}(6,3)^{T} & M_{\tilde{\chi}^{0}}(3,3)
\end{array}\right),
$$

where

$$
M_{\tilde{\chi}^{0}}(6,6)=\left(\begin{array}{cccccc}
M_{1}^{\prime} & 0 & 0 & \Gamma_{H_{1}} & \Gamma_{H_{2}} & \Gamma_{S} \\
0 & M_{1} & 0 & -\frac{1}{\sqrt{2}} g_{1} v_{1} & \frac{1}{\sqrt{2}} g_{1} v_{2} & 0 \\
0 & 0 & M_{2} & \frac{1}{\sqrt{2}} g_{2} v_{1} & -\frac{1}{\sqrt{2}} g_{2} v_{2} & 0 \\
\Gamma_{H_{1}} & -\frac{1}{\sqrt{2}} g_{1} v_{1} & \frac{1}{\sqrt{2}} g_{2} v_{1} & 0 & -h s & -h v_{2} \\
\Gamma_{H_{2}} & \frac{1}{\sqrt{2}} g_{1} v_{2} & -\frac{1}{\sqrt{2}} g_{2} v_{2} & -h s & 0 & -h v_{1} \\
\Gamma_{S} & 0 & 0 & -h v_{2} & -h v_{1} & 0
\end{array}\right),
$$

and

$$
M_{\tilde{\chi}^{0}}(3,3)=\left(\begin{array}{ccc}
0 & -\lambda s_{3} & -\lambda s_{2} \\
-\lambda s_{3} & 0 & -\lambda s_{1} \\
-\lambda s_{2} & -\lambda s_{1} & 0
\end{array}\right),
$$

where $\Gamma_{\phi} \equiv \sqrt{2} g_{Z^{\prime}} Q_{\phi}\langle\phi\rangle$; and $M_{1}^{\prime}, M_{1}, M_{2}$ are gaugino masses for $U(1)^{\prime}, U(1)$ and $S U(2)_{L}$, respectively. The first row of $M_{\tilde{\chi}^{0}}(6,3)$ is given by $\left(\begin{array}{lll}\Gamma_{S_{1}} & \Gamma_{S_{2}} & \Gamma_{S_{3}}\end{array}\right)$, while the other entries are zero.

The chargino mass matrix is

$$
M_{\tilde{\chi}^{ \pm}}=\left(\begin{array}{cc}
M_{2} & \sqrt{2} M_{W} \sin \beta \\
\sqrt{2} M_{W} \cos \beta & \mu
\end{array}\right),
$$

where $\mu \equiv h s$ is the effective $\mu$ parameter.

If $\mu$ is too small the lighter chargino mass will violate observational bounds. However, the Yukawa coupling $h$ (at the electroweak scale) cannot be too large if the theory is to remain perturbative up to a large grand unification or string scale. This constraint is somewhat less restrictive than the corresponding one in the NMSSM [19] because the new contributions from the $U(1)^{\prime}$ to the running of $h$ are negative. We have found that $h$ can be as large as $0.7-0.8$, even for $\tan \beta \sim 1$. We will illustrate the results for the cases $h=0.5$ and $h=0.75$.

For $\lambda \rightarrow 0$ the potential may be unbounded below (depending on the $m_{S_{i}}^{2}$ ) for large $s_{i}$. In that case, for small but finite $\lambda$ the $s_{i}$ will be large, as will the $Z^{\prime}$ mass. We will typically choose $\lambda$ to be around $h / 10$. Though small, $\lambda$ is still much larger than most of the Yukawa couplings associated with the fermion masses.

For $A_{h}$ comparable to the scale of the soft Higgs masses, the potential has an unwanted global minimum at $v_{1}=v_{2}=0$ and $s \sim s_{i}$. This can be avoided by choosing $A_{h}$ to be relatively large, e.g., of order (5-10) larger than the soft masses. In this case, the symmetry breaking is driven more by the $A$ terms than the soft masses, analogous to the large $A$ scenarios described in [16]. In the large $A_{h}$ limit one has 
$s \sim v_{1} \sim v_{2}$. For intermediate $A_{h}$ the ratio of $s / v_{i}$ can be increased to around $3 / 2$, but not much more without introducing the unwanted minimum described above.

Therefore, the lower bound on the light chargino mass from LEP gives a strong constraint on the models. This is in the range $\sim(90-104.5)$ for center mass energy $\sqrt{s}=209 \mathrm{GeV}$, depending on the decay kinematics [20]. Let us discuss the chargino masses in detail. They are 21]

$$
\begin{aligned}
m_{\tilde{\chi}_{1}^{ \pm}}= & \frac{\eta_{C 1}}{2}\left(\sqrt{\left(M_{2}-\mu\right)^{2}+2 M_{W}^{2}(1+\sin 2 \beta)}\right. \\
& \left.-\sqrt{\left(M_{2}+\mu\right)^{2}+2 M_{W}^{2}(1-\sin 2 \beta)}\right), \\
m_{\tilde{\chi}_{2}^{ \pm}}= & \frac{1}{2}\left(\sqrt{\left(M_{2}-\mu\right)^{2}+2 M_{W}^{2}(1+\sin 2 \beta)}\right. \\
& \left.+\sqrt{\left(M_{2}+\mu\right)^{2}+2 M_{W}^{2}(1-\sin 2 \beta)}\right) .
\end{aligned}
$$

where $\eta_{C 1}= \pm 1$ is chosen so that $m_{\tilde{\chi}_{1}^{ \pm}}$is positive. Because $\tan \beta \simeq 1.0$, the light chargino mass is

$$
m_{\tilde{\chi}_{1}^{ \pm}} \simeq \frac{\eta_{C 1}}{2}\left(\sqrt{\left(M_{2}-\mu\right)^{2}+4 M_{W}^{2}}-\left|M_{2}+\mu\right|\right) .
$$

If $M_{2}$ and $\mu$ have the same sign, i.e., $M_{2} \mu>0$, this is typically smaller than $\min \left\{M_{2}, \mu\right\}$. For example, for $|\mu|<\left|M_{2}\right|$ and $\left|M_{2}\right| \gg M_{W}$, one obtains $m_{\tilde{\chi}_{1}^{ \pm}} \sim \mu$. For $M_{2} \mu<0$ one finds $m_{\tilde{\chi}_{1}^{ \pm}}<\sqrt{\mu^{2}+M_{W}^{2}}$. The limit is saturated if and only if $M_{2}=-\mu$, in which case the two chargino masses are equal (for $\tan \beta=1$ ). Thus, for $h<0.8$ and $s / v_{1} \simeq s / v_{2} \lesssim 3 / 2$, the upper bounds on the light chargino mass are around 120 $\mathrm{GeV}$ and $170 \mathrm{GeV}$ for the cases $M_{2} \mu>0$ and $<0$, respectively, with lower values for smaller $h$.

The charged Higgs mass is

$$
M_{H^{ \pm}}^{2}=M_{W}^{2}+\frac{2 A_{h} h s}{\sin 2 \beta}-h^{2}\left(v_{1}^{2}+v_{2}^{2}\right)
$$

where $M_{W}^{2}=\frac{g^{2}}{2}\left(v_{1}^{2}+v_{2}^{2}\right)$.

The upper bound on the tree-level mass of the lightest CP even Higgs doublet scalar, i.e., before including mixing with the $S U(2)$ singlets and corresponding to the lightest scalar $h^{0}$ in the MSSM, is [5]

$$
m_{h^{0}}^{2} \leq M_{Z}^{2} \cos ^{2} 2 \beta+h^{2}\left(v_{1}^{2}+v_{2}^{2}\right) \sin ^{2} 2 \beta+2 g_{Z^{\prime}}^{2} \frac{\left(Q_{H_{1}} v_{1}^{2}+Q_{H_{2}} v_{2}^{2}\right)^{2}}{\left(v_{1}^{2}+v_{2}^{2}\right)} .
$$

In the models considered here, $\tan \beta \simeq 1.0$, so that

$$
m_{h^{0}} \lesssim \sqrt{h^{2}+\frac{1}{2} g_{Z^{\prime}}^{2}\left(Q_{H_{1}}+Q_{H_{2}}\right)^{2}} \times 174 \mathrm{GeV},
$$


which is much weaker than the corresponding limit $m_{h^{0}}<M_{Z}$ in the MSSM. Of course, the actual Higgs mass eigenstates involve mixing of the doublets with $S U(2)$ singlets, so that the tree-level mass eigenvalues are lower. Also, one must add poten-

tially large loop corrections in both cases. The coefficient $\sqrt{h^{2}+\frac{1}{2} g_{Z^{\prime}}^{2}\left(Q_{H_{1}}+Q_{H_{2}}\right)^{2}}$ is typically of order unity.

It is still necessary to have two off-diagonal dimension-2 soft supersymmetry breaking terms involving $S, S_{1}, S_{2}$ and $S_{3}$ to break the two unwanted global $U(1)$ symmetries. We cannot choose $\left|Q_{S_{1}}\right| \neq\left|Q_{S_{2}}\right| \neq\left|Q_{S_{3}}\right|$ because then at most one term, $S S_{i}$ or $S S_{i}^{\dagger}$, would be allowed. The only possibilities are $Q_{S_{1}}=Q_{S_{2}}=-\frac{1}{2} Q_{S_{3}}$ and $Q_{S_{1}}=-Q_{S_{2}}, Q_{S_{3}}=0$. Any $\mathrm{VEV}$ of $S_{3}$ in the second case would not be linked to $U(1)^{\prime}$ breaking; we will not consider this possibility further. In the first case, there are two possibilities for the $U(1)^{\prime}$ charge of $S: Q_{S}= \pm Q_{S_{1}}$ and $Q_{S}= \pm Q_{S_{3}}$, which will be discussed as Model I and Model II, respectively[?

Further details of the Higgs masses and eigenstates are given in the Appendices.

\section{Specific Models}

\subsection{Model I}

In Model I, we choose the $U(1)^{\prime}$ charges for the Higgs fields as

$$
Q_{S}=-Q_{S_{1}}=-Q_{S_{2}}=\frac{1}{2} Q_{S_{3}}, Q_{H_{1}}+Q_{H_{2}}+Q_{S}=0
$$

The dimension-2 supersymmetry breaking soft terms

$$
V_{\text {soft }}^{(I)}=\left(m_{S S_{1}}^{2} S S_{1}+m_{S S_{2}}^{2} S S_{2}+m_{S_{1} S_{2}}^{2} S_{1}^{\dagger} S_{2}+\text { H.C. }\right)
$$

are allowed by the $U(1)^{\prime}$, so in general

$$
V_{\text {soft }}=V_{\text {soft }}^{(a)}+V_{\text {soft }}^{(I)}
$$

where $V_{\text {soft }}^{(a)}$ is defined in $(\mathbb{\Psi})$. However, only two of these are needed to break the global $U(1)$ symmetries, so for simplicity we will setf $m_{S_{1} S_{2}}^{2}=0$.

\footnotetext{
${ }^{3}$ These charges allow additional superpotential terms $S_{1}^{2} S_{3}$ and $S_{2}^{2} S_{3}$. Their presence would have little effect on our conclusions, other than changing the relative sizes of the $s_{i}$, so they will be ignored for simplicity. They could also be explicitly eliminated by discrete symmetries for the dimension- 4 operators, or by string selection rules if there is an underlying string theory. Similarly, the $U(1)^{\prime}$ symmetry would allow additional terms $S S_{1,2}$ (Model I) or $S S_{3}$ (Model II) in the superpotential. These again would not affect our conclusions if present, and in any case bilinear terms are not expected in (conformal) string theory.

${ }^{4}$ Keeping a nonzero $m_{S_{1} S_{2}}^{2}$ would yield a spectrum similar to Model II. In the most general case one would have to allow $m_{S_{1} S_{2}}^{2}$ to be complex valued (and therefore CP violating) because there would not be enough freedom of field redefinitions to ensure that all three terms are real and negative.
} 
require

To avoid directions of the potential that are not bounded from below, we

$$
m_{S}^{2}+m_{S_{1}}^{2}+2 m_{S S_{1}}^{2}>0, m_{S}^{2}+m_{S_{2}}^{2}+2 m_{S S_{2}}^{2}>0
$$

The first condition corresponds to the direction in which $s=s_{1}$ with the other VEVs vanishing, for which the quartic and cubic terms in the potential are flat. The second corresponds to $s=s_{2}$.

The minimization conditions for the neutral scalar potential with non-zero VEVs, and the mass matrices for the CP odd and even Higgses are given in Appendix A.

\subsection{Model II}

In Model II, we choose the $U(1)^{\prime}$ charges

$$
Q_{S_{1}}=Q_{S_{2}}=\frac{1}{2} Q_{S}=-\frac{1}{2} Q_{S_{3}}, Q_{H_{1}}+Q_{H_{2}}+Q_{S}=0
$$

allowing the dimension- 2 supersymmetry terms

$$
V_{\text {soft }}^{(I I)}=\left(m_{S S_{3}}^{2} S S_{3}+m_{S_{1} S_{2}}^{2} S_{1}^{\dagger} S_{2}+\text { H.C. }\right)
$$

so that

$$
V_{\text {soft }}=V_{\text {soft }}^{(a)}+V_{\text {soft }}^{(I I)}
$$

To avoid unbounded from below directions, we require

$$
m_{S}^{2}+2 m_{S_{1}}^{2}>0, m_{S}^{2}+2 m_{S_{2}}^{2}>0
$$

corresponding to the directions with $s_{1}=\sqrt{2} s$ and $s_{2}=\sqrt{2} s$ (and the other VEVs zero), respectively.

The minimization conditions and mass matrices are similar to those in Model I up to obvious changes, so they will not be repeated.

\subsection{Numerical Results for Some Particle Spectra}

In this subsection, we present the numerical results for the $Z^{\prime}$ boson mass; the $Z-Z^{\prime}$ mixing angle $\alpha_{Z-Z^{\prime}}$; and the chargino, neutralino, and Higgs masses for the two models. To generate the mass hierarchy between the $Z$ and $Z^{\prime}$, we choose $\lambda=$ $h / 10$. We illustrate for two values of $h$, i.e., 0.5 and 0.75 . Both are theoretically and phenomenologically acceptable. However, the larger value allows larger chargino and neutralino masses, but is close to the upper limit allowed if the theory is to remain perturbative to a large scale. In our conventions, $\mu>0$, while the gaugino masses $M_{i}$ can be positive or negative. We choose two examples, i.e., $M_{1}=-100$ 
Table 1: $v_{1}, v_{2}, s, s_{1}, s_{2}, s_{3}$; the $Z$ and $Z^{\prime}$ masses; and $\alpha_{Z-Z^{\prime}}$ in Models I and II. The masses and VEVs are in GeV.

\begin{tabular}{|c|c|c|c|c|c|c|c|c|c|c|}
\hline Model & $h$ & $v_{1}$ & $v_{2}$ & $s$ & $s_{1}$ & $s_{2}$ & $s_{3}$ & $Z$ & $Z^{\prime}$ & $\alpha_{Z-Z^{\prime}}$ \\
\hline I & 0.5 & 121 & 125 & 187 & 1270 & 1260 & 1260 & 91 & 2030 & $3.8 \times 10^{-3}$ \\
\hline I & 0.75 & 121 & 125 & 187 & 1270 & 1260 & 1260 & 91 & 2030 & $3.8 \times 10^{-3}$ \\
\hline II & 0.5 & 122 & 124 & 175 & 1300 & 1300 & 1290 & 91 & 2100 & $4.7 \times 10^{-3}$ \\
\hline II & 0.75 & 122 & 124 & 178 & 1310 & 1310 & 1300 & 91 & 2110 & $4.7 \times 10^{-3}$ \\
\hline
\end{tabular}

$\mathrm{GeV}, M_{2}=-200 \mathrm{GeV}$ and $M_{1}^{\prime}=-600 \mathrm{GeV}$; and $M_{1}=200 \mathrm{GeV}, M_{2}=400 \mathrm{GeV}$ and $M_{1}^{\prime}=600 \mathrm{GeV}$. These choices can yield relatively large masses for the lighter charginos. We also choose the standard GUT value $g_{Z^{\prime}}=\sqrt{5 / 3} g_{1}$ (it is $\sqrt{5 / 3} g_{1}$ that unifies with $g_{2}$ and $g_{3}$ in the simple GUT models). This is for illustration only; we do not insist on conventional grand unificationf. As described above, we choose large values for $A_{h}$ (and also choose $A_{\lambda} \sim A_{h}$ ). Otherwise, the minimum of the potential would be for $v_{1}=v_{2}=0$ and $s \sim s_{i}$. (Even for large $A_{h}$ there is such a local minimum. However, there is also the desired $S U(2)$-breaking minimum closer to the origin.) The terms linear in $s$ in $V_{\text {soft }}^{(I, I I)}$ and the $A_{h}$ term prevent unphysical minima such as $s=v_{1}=0, s=v_{2}=0$, or only one of the three vanishing.

The input parameters with dimensions or mass of mass-squared are chosen in arbitrary units. After finding an acceptable minimum they are rescaled so that $\sqrt{v_{1}^{2}+v_{2}^{2}} \simeq 174 \mathrm{GeV}$. For Model I, we choose: $A_{h}=A_{\lambda}=1.0, m_{H_{1}}^{2}=m_{H_{2}}^{2}=m_{S}^{2}=$ $-0.010, m_{S_{1}}^{2}=m_{S_{2}}^{2}=0.031, m_{S_{3}}^{2}=-0.010, m_{S S_{1}}^{2}=m_{S S_{2}}^{2}=-0.010, Q_{H_{1}}=1, Q_{H_{2}}=$ $-2, Q_{S}=-Q_{S_{1}}=-Q_{S_{2}}=1$, and $Q_{S_{3}}=2$.

For $h=0.5$ and $\lambda=0.05$ the VEVs at the minimum are $v_{1}=0.928, v_{2}=$ $0.953, s=1.43, s_{1}=9.67, s_{2}=9.65$, and $s_{3}=9.63$. For $h=0.75$ and $\lambda=0.075$ they are $v_{1}=0.616, v_{2}=0.636, s=0.953, s_{1}=6.44, s_{2}=6.42$, and $s_{3}=6.41$. The rescaled VEVs and the corresponding $Z^{\prime}$ mass and $Z-Z^{\prime}$ mixing angle are listed in Table 1.

For Model II, we choose $A_{h}=A_{\lambda}=1.0, m_{H_{1}}^{2}=m_{H_{2}}^{2}=-0.010, m_{S}^{2}=$ $-0.020, m_{S_{1}}^{2}=m_{S_{2}}^{2}=0.011, m_{S_{3}}^{2}=-0.010, m_{S S_{3}}^{2}=m_{S_{1} S_{2}}^{2}=-0.015, Q_{H_{1}}=1, Q_{H_{2}}=$ $-3, Q_{S}=2, Q_{S_{1}}=Q_{S_{2}}=1, Q_{S_{3}}=-2$. The VEVs are $v_{1}=0.955, v_{2}=0.965, s=$ $1.37, s_{1}=10.1, s_{2}=10.1, s_{3}=10.1$ for $h=0.5$ and $\lambda=0.05$; and $v_{1}=0.632, v_{2}=$ $0.638, s=0.919, s_{1}=6.76, s_{2}=6.75, s_{3}=6.73$ for $h=0.75$ and $\lambda=0.075$.

The rescaled VEVs $v_{1}, v_{2}, s, s_{1}, s_{2}, s_{3}$; the mixing $\alpha_{Z-Z^{\prime}}$; and the particle spectra are given in Tables 1, 2, and 3. It is seen that the two models yield similar

\footnotetext{
${ }^{5}$ Many unification models would suggest $M_{1}^{\prime} \sim M_{1}$. The only effect of a smaller $M_{1}^{\prime}$ would be small changes in the spectrum of the neutralinos in the secluded sector.
} 
Table 2: The chargino and neutralino masses in GeV for Models I and II.

\begin{tabular}{|c|c|c|c|c|c|c|c|c|c|c|c|c|c|}
\hline Model & $h$ & $M_{i}$ & $\tilde{\chi}_{1}^{ \pm}$ & $\tilde{\chi}_{2}^{ \pm}$ & $\tilde{\chi}_{1}^{0}$ & $\tilde{\chi}_{2}^{0}$ & $\tilde{\chi}_{3}^{0}$ & $\tilde{\chi}_{4}^{0}$ & $\tilde{\chi}_{5}^{0}$ & $\tilde{\chi}_{6}^{0}$ & $\tilde{\chi}_{7}^{0}$ & $\tilde{\chi}_{8}^{0}$ & $\tilde{\chi}_{9}^{0}$ \\
\hline I & 0.5 & $<0$ & 114 & 220 & 52 & 63 & 107 & 122 & 126 & 145 & 221 & 1790 & 2320 \\
\hline I & 0.5 & $>0$ & 74 & 420 & 52 & 61 & 63 & 126 & 145 & 213 & 420 & 1710 & 2380 \\
\hline I & 0.75 & $<0$ & 158 & 218 & 78 & 94 & 106 & 165 & 189 & 218 & 219 & 1800 & 2310 \\
\hline I & 0.75 & $>0$ & 118 & 423 & 78 & 94 & 100 & 189 & 217 & 218 & 423 & 1700 & 2390 \\
\hline II & 0.5 & $<0$ & 108 & 221 & 54 & 65 & 107 & 116 & 130 & 141 & 222 & 1860 & 2390 \\
\hline II & 0.5 & $>0$ & 68 & 419 & 54 & 56 & 65 & 130 & 141 & 212 & 420 & 1780 & 2450 \\
\hline II & 0.75 & $<0$ & 152 & 218 & 80 & 98 & 106 & 158 & 196 & 213 & 219 & 1890 & 2390 \\
\hline II & 0.75 & $>0$ & 111 & 422 & 80 & 94 & 98 & 196 & 213 & 216 & 423 & 1780 & 2480 \\
\hline
\end{tabular}

spectra, since each is $A_{h}$ dominated. The composition of the physical mass eigenstates in terms of the weak eigenstates are given for Model I in Appendix B. The spectra are quite different from the MSSM. The most important feature is that the VEVs of the secluded sector fields $\left(S_{1}, S_{2}\right.$, and $\left.S_{3}\right)$ are much larger than those of the ordinary sector $\left(H_{i}\right.$ and $\left.S\right)$, without any fine tuning of parameters. This leads to a rather heavy $Z^{\prime}$, a small $\alpha_{Z-Z^{\prime}}\left(\alpha_{z-z^{\prime}}\right.$ would have been zero or extremely small, depending on the soft mass-squares, if we had chosen $Q_{H_{1}}=Q_{H_{2}}$ ), and little mixing between the ordinary and secluded sectors. The large $A_{h}$ needed to ensure the correct vacuum implies $\tan \beta \sim 1$ and $s / v_{i} \lesssim 3 / 2$, leading to significant mixing between the doublet and singlet Higgs fields, and also between the corresponding neutralinos.

The upper limit on the lightest CP even Higgs doublet particle is considerably relaxed compared to the MSSM and even the NMSSM. However, the actual mass eigenvalues are reduced by mixing with the $S U(2)$ singlet. For example, in Model I with $h=0.5$, the lightest scalar, $H_{1}^{0}$, and $H_{4}^{0}$ are roughly equal admixtures of singlet $(S)$ and doublets; $H_{5}^{0}$ is almost pure doublet; while the two light states $H_{2,3}^{0}$ and the very heavy $H_{6}^{0}$ consist almost entirely of the secluded fields. Similarly, the CP odd states $A_{2}^{0}$ and $A_{4}^{0}$ are ordinary-sector doublet-singlet mixtures, while the very light $A_{1}^{0}$ and the heavier $A_{3}^{0}$ consist mainly of the $S_{i}$. (The small values for the $A_{1}^{0}$ mass reflect the fact that the off-diagonal scalar masses added to break the two global symmetries were chosen to be small compared to $A_{h}$ and $A_{\lambda}$, and, in the case of Model I, that the terms involve $s \ll s_{1,2}$.) The lightest $\mathrm{CP}$ even Higgs has tree-level mass $\sim 52 \mathrm{GeV}$ for Model I with $h=0.5$. It is not clear whether this is consistent with experiment. In the first place, the masses may increase significantly due to radiative corrections analogous to those of the MSSM. However, the actual values depend on parameters 
Table 3: The charged, CP even, and CP odd Higgs masses in GeV at tree-level for Models I and II.

\begin{tabular}{|c|c|c|c|c|c|c|c|c|c|c|c|c|}
\hline Model & $h$ & $H^{ \pm}$ & $H_{1}^{0}$ & $H_{2}^{0}$ & $H_{3}^{0}$ & $H_{4}^{0}$ & $H_{5}^{0}$ & $H_{6}^{0}$ & $A_{1}^{0}$ & $A_{2}^{0}$ & $A_{3}^{0}$ & $A_{4}^{0}$ \\
\hline I & 0.5 & 152 & 52 & 88 & 92 & 112 & 158 & 2030 & 5.0 & 43 & 157 & 174 \\
\hline I & 0.75 & 211 & 78 & 131 & 139 & 168 & 215 & 2030 & 7.6 & 65 & 236 & 261 \\
\hline II & 0.5 & 146 & 59 & 92 & 93 & 108 & 152 & 2100 & 22 & 38 & 158 & 168 \\
\hline II & 0.75 & 203 & 86 & 139 & 140 & 162 & 207 & 2120 & 34 & 58 & 239 & 255 \\
\hline
\end{tabular}

associated with the sfermion sector of the model, which we are not considering here. Also, the state involves a large admixture of $S U(2)$ singlet, so the usual SM and MSSM limits do not apply directly, and will require a detailed study of the collider implications of such mixings that is beyond the scope of this paper. Of course, the mass could be increased somewhat for different choices of the soft parameters.

The chargino masses are consistent with the experimental limits except for the cases with $h=0.5$ and $M_{2}>0$. The lighter chargino is dominantly Higgsino for our choices of $M_{2}$, because of the relatively low effective $\mu$ parameter.

In the first row of Table 2, the lightest neutralino $\tilde{\chi}_{1}^{0}$ is mainly singlino $\tilde{S}$, with a nontrivial admixture of Higgsino. This is somewhat similar to the model in [22], in which the light singlino was advocated as a dark matter candidate. $\tilde{\chi}_{6}^{0}$ is also a mixture, while $\tilde{\chi}_{4}^{0}, \tilde{\chi}_{3}^{0}$, and $\tilde{\chi}_{7}^{0}$ are mainly Higgsino, bino, and wino, respectively. The actual composition of these ordinary sector states is affected by the low effective $\mu$, but also depends significantly on the choice of gaugino mass inputs. $\tilde{\chi}_{2}^{0}$ and $\tilde{\chi}_{5}^{0}$ are mainly secluded sector singlinos, while the two heavy states $\tilde{\chi}_{8}^{0}$ and $\tilde{\chi}_{9}^{0}$ are admixtures of $\tilde{B}^{\prime}$ and singlino $\left(\tilde{S}_{i}\right)$.

The soft masses for squarks and sleptons are independent of the symmetry breaking sector. However, if they are chosen to be of the same order as $A_{h}$ (i.e., larger than the soft masses of the doublet and singlet Higgs fields) then they would typically be in the $100-300 \mathrm{GeV}$ range.

Clearly, the spectrum of the symmetry breaking sector is very rich, and will differ significantly from that of the MSSM because of the significant doublet-singlet mixing. A detailed study of the collider signatures and limits and the implications for cold dark matter is beyond the scope of this paper. However, there will generically be a number of particles associated with the symmetry breaking sector that are on the margin of being excluded or discovered. A further study would be very interesting. 


\section{Discussion and Conclusion}

Many theories beyond the Standard Model predict the existence of addition $U(1)^{\prime}$ gauge symmetries broken near the electroweak scale. Although such models have the desirable feature of yielding a simple solution to the $\mu$ problem, they suffer from the need to make the $Z^{\prime}$ sufficiently heavy, typically at least (500-800) GeV. Previous models have often assumed that this is accomplished by having a typical soft supersymmetry breaking scale (and corresponding sfermion masses) of a $\mathrm{TeV}$ or so, with the electroweak scale smaller by cancellations. In this paper, we present a different mechanism, in which the large $U(1)^{\prime}$ breaking scale is associated with an almost flat direction of the quartic terms of the scalar potential. In the limit that a certain Yukawa coupling goes to zero, this could correspond to a runaway, unbounded from below direction. The flatness is lifted by small but nonzero values, allowing a large $Z^{\prime} / Z$ mass hierarchy and a small (or almost zero for some charge assignments) $Z-Z^{\prime}$ mixing angle.

We have presented examples of such models involving an ordinary sector of symmetry breaking fields, which includes two Higgs doublets and an $S U(2)$ singlet $S$ which generates an effective $\mu$ parameter; and a secluded sector involving three $S U(2)$ singlet fields $S_{i}, i=1,2,3$, which acquire large VEVs. The two sectors are only weakly coupled by $U(1)^{\prime}$ interactions and soft scalar mass terms. The ordinary sector is somewhat similar to the NMSSM, but involves parameter choices very different from those usually studied in the NMSSM context. (These include the absence of a cubic term in $S$ in the superpotential, a larger allowed value for $h$, and a large $A_{h}$.) We carried out a detailed study of such issues as unwanted global minima and runaway directions, unwanted global symmetries, the upper limit from perturbative unification on the Yukawa coupling associated with the effective $\mu$ parameter, and the need to have sufficiently heavy charginos. Acceptable parameter ranges were found, characterized by: the electroweak symmetry breaking is driven more by a large $A$ term than by the soft scalar mass-squares, leading to $\tan \beta \sim 1$; a VEV of $S$ comparable to the electroweak scale; a fairly small effective $\mu$ parameter (typically $80-140 \mathrm{GeV}$ ); and a much larger $U(1)^{\prime}$ breaking scale generated by the VEVs of the $S_{i}$.

The spectrum of the symmetry breaking sector is very rich. There are a number of light CP even and odd Higgs fields and neutralinos, for example, which involve significant mixing between $S U(2)$ doublet and singlet fields. A detailed study of the implications for colliders and cosmology is beyond the scope of this paper, but it is expected that a number of the predicted states are close to being excluded or discovered.

We have also not attempted to embed the models into a full theory. This would be necessary to discuss the sfermion spectrum; the cancellation of anomalies; possible flavor changing effects [23]; and some aspects of the production and decay of the Higgs particles, charginos, and neutralinos. 


\section{Acknowledgments}

This research was supported in part by the U.S. Department of Energy under Grants No. DOE-EY-76-02-3071 and DE-FG02-95ER40896, and by the University of Wisconsin at Madison.

\section{Appendix A}

In Appendix A we discuss the minimization conditions and scalar mass matrices for Model I. The conditions for Model II are similar.

The potential minimization conditions for the neutral scalar fields with nonzero VEVs are

$$
\begin{gathered}
m_{H_{1}}^{2}-A_{h} h s v_{2} / v_{1}+h^{2}\left(v_{2}^{2}+s^{2}\right)+\frac{G^{2}}{4}\left(v_{1}^{2}-v_{2}^{2}\right)+g_{Z^{\prime}}^{2} Q_{H_{1}} \Delta=0, \\
m_{H_{2}}^{2}-A_{h} h s v_{1} / v_{2}+h^{2}\left(v_{1}^{2}+s^{2}\right)+\frac{G^{2}}{4}\left(v_{2}^{2}-v_{1}^{2}\right)+g_{Z^{\prime}}^{2} Q_{H_{2}} \Delta=0, \\
m_{S}^{2}-A_{h} h v_{1} v_{2} / s+m_{S S_{1}}^{2} s_{1} / s+m_{S S_{2}}^{2} s_{2} / s+h^{2}\left(v_{1}^{2}+v_{2}^{2}\right)+g_{Z^{\prime}}^{2} Q_{S} \Delta=0, \\
m_{S_{1}}^{2}-A_{\lambda} \lambda s_{2} s_{3} / s_{1}+m_{S S_{1}}^{2} s / s_{1}+\lambda^{2}\left(s_{2}^{2}+s_{3}^{2}\right)+g_{Z^{\prime}}^{2} Q_{S_{1}} \Delta=0, \\
m_{S_{2}}^{2}-A_{\lambda} \lambda s_{1} s_{3} / s_{2}+m_{S S_{2}}^{2} s / s_{2}+\lambda^{2}\left(s_{1}^{2}+s_{3}^{2}\right)+g_{Z^{\prime}}^{2} Q_{S_{2}} \Delta=0, \\
m_{S_{3}}^{2}-A_{\lambda} \lambda s_{1} s_{2} / s_{3}+\lambda^{2}\left(s_{1}^{2}+s_{2}^{2}\right)+g_{Z^{\prime}}^{2} Q_{S_{3}} \Delta=0 .
\end{gathered}
$$

The mass-square matrix for the CP odd neutral Higgs particles in the basis $\left\{H_{1}^{0 i} \equiv \sqrt{2} \operatorname{Im}\left(H_{1}^{0}\right), H_{2}^{0 i}, S^{0 i}, S_{1}^{0 i}, S_{2}^{0 i}, S_{3}^{0 i}\right\}$ is

$$
M_{A^{0}}^{2}=\left(\begin{array}{cc}
O_{A^{0}} & C_{A^{0}} \\
C_{A^{0}}^{T} & S_{A^{0}}
\end{array}\right)
$$

where

$$
\begin{aligned}
O_{A^{0}}= & \left(\begin{array}{ccc}
A_{h} h s v_{2} / v_{1} & A_{h} h s & A_{h} h v_{2} \\
A_{h} h s & A_{h} h s v_{1} / v_{2} & A_{h} h v_{1} \\
A_{h} h v_{2} & A_{h} h v_{1} & \beta_{S}^{2}
\end{array}\right), \\
S_{A^{0}} & =\left(\begin{array}{ccc}
\beta_{S_{1}}^{2} & A_{\lambda} \lambda s_{3} & A_{\lambda} \lambda s_{2} \\
A_{\lambda} \lambda s_{3} & \beta_{S_{2}}^{2} & A_{\lambda} \lambda s_{1} \\
A_{\lambda} \lambda s_{2} & A_{\lambda} \lambda s_{1} & A_{\lambda} \lambda s_{1} s_{2} / s_{3}
\end{array}\right),
\end{aligned}
$$




$$
C_{A^{0}}=\left(\begin{array}{ccc}
0 & 0 & 0 \\
0 & 0 & 0 \\
-m_{S S_{1}}^{2} & -m_{S S_{2}}^{2} & 0
\end{array}\right)
$$

and

$$
\begin{gathered}
\beta_{S}^{2}=\left(A_{h} h v_{1} v_{2}-m_{S S_{1}}^{2} s_{1}-m_{S S_{2}}^{2} s_{2}\right) / s, \\
\beta_{S_{1}}^{2}=A_{\lambda} \lambda s_{2} s_{3} / s_{1}-m_{S S_{1}}^{2} s / s_{1}, \\
\beta_{S_{2}}^{2}=A_{\lambda} \lambda s_{1} s_{3} / s_{2}-m_{S S_{2}}^{2} s / s_{2} .
\end{gathered}
$$

Similarly, in the basis $\left\{H_{1}^{0 r} \equiv \sqrt{2} R e\left(H_{1}^{0}\right), H_{2}^{0 r}, S^{0 r}, S_{1}^{0 r}, S_{2}^{0 r}, S_{3}^{0 r}\right\}$, the masssquare matrix for the $\mathrm{CP}$ even neutral Higgs particles is

$$
M_{H^{0}}^{2}=\left(\begin{array}{ll}
O_{H^{0}} & C_{H^{0}} \\
C_{H^{0}}^{T} & S_{H^{0}}
\end{array}\right)
$$

where

$$
\begin{gathered}
O_{H^{0}}=\left(\begin{array}{ccc}
\kappa_{H_{1}}^{2} & \kappa_{H_{1}, H_{2}} & \kappa_{H_{1}, S} \\
\kappa_{H_{1}, H_{2}} & \kappa_{H_{2}}^{2} & \kappa_{H_{2}, S} \\
\kappa_{H_{1}, S} & \kappa_{H_{2}, S} & \kappa_{S}^{2}
\end{array}\right), \\
S_{H^{0}}=\left(\begin{array}{ccc}
\kappa_{S_{1}}^{2} & \kappa_{S_{1}, S_{2}} & \kappa_{S_{1}, S_{3}} \\
\kappa_{S_{1}, S_{2}} & \kappa_{S_{2}}^{2} & \kappa_{S_{2}, S_{3}} \\
\kappa_{S_{1}, S_{3}} & \kappa_{S_{2}, S_{3}} & \kappa_{S_{3}}^{2}
\end{array}\right), \\
C_{H^{0}}=\left(\begin{array}{ccc}
\kappa_{H_{1}, S_{1}} & \kappa_{H_{1}, S_{2}} & \kappa_{H_{1}, S_{3}} \\
\kappa_{H_{2}, S_{1}} & \kappa_{H_{2}, S_{2}} & \kappa_{H_{2}, S_{3}} \\
\kappa_{S, S_{1}}+m_{S, S_{1}}^{2} & \kappa_{S, S_{2}}+m_{S, S_{2}}^{2} & \kappa_{S, S_{3}}
\end{array}\right),
\end{gathered}
$$

and

$$
\begin{gathered}
\kappa_{H_{i}}^{2}=2\left(\frac{G^{2}}{4}+g_{Z^{\prime}}^{2} Q_{H_{i}}^{2}\right) v_{i}^{2}+A_{h} h s v_{1} v_{2} / v_{i}^{2}, \\
\kappa_{S}^{2}=2 g_{Z^{\prime}}^{2} Q_{S^{\prime}}^{2} s^{2}+\left(A_{h} h v_{1} v_{2}-m_{S S_{1}}^{2} s_{1}-m_{S S_{2}}^{2} s_{2}\right) / s, \\
\kappa_{S_{1}}^{2}=2 g_{Z^{\prime}}^{2} Q_{S_{1}}^{2} s_{1}^{2}+A_{\lambda} \lambda s_{2} s_{3} / s_{1}-m_{S S_{1}}^{2} s / s_{1}, \\
\kappa_{S_{2}}^{2}=2 g_{Z^{\prime}}^{2} Q_{S_{2}}^{2} s_{2}^{2}+A_{\lambda} \lambda s_{1} s_{3} / s_{2}-m_{S S_{2}}^{2} s / s_{2},
\end{gathered}
$$




$$
\begin{gathered}
\kappa_{S_{3}}^{2}=2 g_{Z^{\prime}}^{2} Q_{S_{3}}^{2} s_{3}^{2}+A_{\lambda} \lambda s_{1} s_{2} / s_{3}, \\
\kappa_{H_{1}, H_{2}}=2\left(h^{2}-\frac{G^{2}}{4}+g_{Z^{\prime}}^{2} Q_{H_{1}} Q_{H_{2}}\right) v_{1} v_{2}-A_{h} h s \\
\kappa_{H_{i}, S}=2\left(h^{2}+g_{Z^{\prime}}^{2} Q_{H_{i}} Q_{S}\right) v_{i} s-\left|\epsilon_{i j}\right| A_{h} h v_{j}, \\
\kappa_{H_{i}, S_{j}}=2 g_{Z^{\prime}}^{2} Q_{H_{i}} Q_{S_{j}} v_{i} s_{j}, \kappa_{S, S_{i}}=2 g_{Z^{\prime}}^{2} Q_{S} Q_{S_{i}} s s_{i}, \\
\kappa_{S_{i}, S_{j}}=2\left(\lambda^{2}+g_{Z^{\prime}}^{2} Q_{S_{i}} Q_{S_{j}}\right) s_{i} s_{j}-\left|\epsilon_{i j k}\right| A_{\lambda} \lambda s_{k} .
\end{gathered}
$$

The upper limit in (21) on the lightest doublet Higgs mass is obtained from the limit on the smaller eigenvalue of the upper $2 \times 2$ sub-block of $O_{H^{0}}$.

\section{Appendix B}

The chargino mass terms are 21]

$$
\mathcal{L}=-\left(\psi^{-}\right)^{T} M_{\tilde{\chi}^{ \pm}} \psi^{+}+\text {H.C. }
$$

where $\left(\psi^{+}\right)^{T}=\left(-i \tilde{W}^{+}, \tilde{H}_{2}^{+}\right)$and $\left(\psi^{-}\right)^{T}=\left(-i \tilde{W}^{-}, \tilde{H}_{1}^{-}\right)$are two component spinors, and $M_{\tilde{\chi}^{ \pm}}$is given in Eq. (16). The chargino mass eigenstates are defined by

$$
\tilde{\chi}_{i}^{+}=V_{i j} \psi_{j}^{+} ; \tilde{\chi}_{i}^{-}=U_{i j} \psi_{j}^{-},
$$

where $U$ and $V$ are unitary matrices.

The eigenvectors for the charginos; neutralinos; and CP even and odd Higgses in Model I with $h=0.5$ and $h=0.75$ are given in Tables 412 .

\section{References}

[1] M. Cvetič and P. Langacker, Phys. Rev. D 54, 3570 (1996) and Mod. Phys. Lett. A 11, 1247 (1996).

[2] For a review, see, M. Cvetič and P. Langacker, in Perspectives on supersymmetry, ed. G. L. Kane (World, Singapore, 1998), p. 312.

[3] For a review, see C. T. Hill and E. H. Simmons, hep-ph/0203079.

[4] D. Suematsu and Y. Yamagishi, Int. J. Mod. Phys. A 10, 4521 (1995). 
Table 4: The eigenvectors for the charginos in Model I.

\begin{tabular}{|c|c|c|c|c|c|c|c|c|c|}
\hline$h$ & $M_{i}$ & $U_{11}$ & $U_{12}$ & $U_{21}$ & $U_{22}$ & $V_{11}$ & $V_{12}$ & $V_{21}$ & $V_{22}$ \\
\hline 0.5 & $<0$ & 0.237 & 0.971 & 0.971 & -0.237 & 0.257 & 0.967 & -0.967 & 0.257 \\
\hline 0.5 & $>0$ & -0.236 & 0.972 & 0.972 & 0.236 & 0.240 & -0.971 & 0.971 & 0.240 \\
\hline 0.75 & $<0$ & 0.197 & 0.980 & 0.980 & -0.197 & 0.238 & 0.971 & -0.971 & 0.238 \\
\hline 0.75 & $>0$ & -0.270 & 0.963 & 0.963 & 0.270 & 0.275 & -0.962 & 0.962 & 0.275 \\
\hline
\end{tabular}

[5] M. Cvetič, D. A. Demir, J. R. Espinosa, L. L. Everett and P. Langacker, Phys. Rev. D 56, 2861 (1997) [Erratum-ibid. D 58, 119905 (1997)]. For the case of gauge mediated symmetry breaking, see P. Langacker, N. Polonsky and J. Wang, Phys. Rev. D 60, 115005 (1999).

[6] J. Ellis, J. F. Gunion, H. E. Haber, L. Roszkowski, and F. Zwirner, Phys. Rev. D 39, 844 (1989); and references therein. Recent references may be found in U. Ellwanger, J. F. Gunion, and C. Hugonie, hep-ph/0111179.

[7] L. Durand and J. L. Lopez, Phys. Lett. B 217, 463 (1989); M. Drees, Int. J. Mod. Phys. A 4, 3635 (1989); M. Cvetič et al, ref. [5]. Other references are given in [2].

[8] J. Erler, Nucl. Phys. B 586, 73 (2000).

[9] G. Cleaver, M. Cvetič, J. R. Espinosa, L. L. Everett and P. Langacker, Phys. Rev. D 57, 2701 (1998).

[10] For reviews, see M. Cvetič and S. Godfrey, hep-ph/9504216; A. Leike, Phys. Rept. 317, 143 (1999).

[11] F. Abe et al. [CDF Collaboration], Phys. Rev. Lett. 79, 2192 (1997).

[12] J. Erler and P. Langacker, Phys. Lett. B 456, 68 (1999), and references therein.

[13] G. P. Zeller et al. [NuTeV Collaboration], Phys. Rev. Lett. 88, 091802 (2002).

[14] C.S. Wood et al., Science 275, 1759 (1997); S.C. Bennett and C.E. Wieman, Phys. Rev. Lett. 82, 2484 (1999); V.A. Dzuba, V.V. Flambaum, and J.S.M. Ginges, hep-ph/0204134.

[15] R. Casalbuoni, S. De Curtis, D. Dominici and R. Gatto, Phys. Lett. B 460, 135 (1999); J. L. Rosner, Phys. Rev. D 61, 016006 (2000); J. Erler and P. Langacker, Phys. Rev. Lett. 84, 212 (2000). 
Table 5: The eigenvectors for the neutralinos in Model I with $h=0.5$ and $M_{i}<0$.

\begin{tabular}{|c|c|c|c|c|c|c|c|c|c|}
\hline Fields & $\tilde{\chi}_{1}^{0}$ & $\tilde{\chi}_{2}^{0}$ & $\tilde{\chi}_{3}^{0}$ & $\tilde{\chi}_{4}^{0}$ & $\tilde{\chi}_{5}^{0}$ & $\tilde{\chi}_{6}^{0}$ & $\tilde{\chi}_{7}^{0}$ & $\tilde{\chi}_{8}^{0}$ & $\tilde{\chi}_{9}^{0}$ \\
\hline$\tilde{B}^{\prime}$ & 0.0 & 0.0 & 0.001 & 0.002 & 0.0 & 0.001 & -0.003 & -0.647 & 0.762 \\
\hline$\tilde{B}$ & -0.004 & 0.0 & 0.978 & 0.188 & 0.0 & -0.007 & -0.093 & 0.002 & -0.001 \\
\hline$\tilde{W}_{3}^{0}$ & 0.005 & 0.0 & 0.137 & -0.237 & 0.0 & -0.010 & 0.962 & -0.003 & 0.002 \\
\hline$\tilde{H}_{1}^{0}$ & -0.356 & 0.0 & 0.116 & -0.675 & 0.0 & 0.610 & -0.174 & -0.030 & -0.025 \\
\hline$\tilde{H}_{2}^{0}$ & -0.368 & 0.0 & -0.108 & 0.668 & 0.0 & 0.604 & 0.188 & 0.062 & 0.051 \\
\hline$\tilde{S}$ & 0.856 & 0.001 & 0.005 & 0.015 & 0.0 & 0.513 & 0.004 & -0.045 & -0.039 \\
\hline$\tilde{S}_{1}$ & 0.028 & -0.707 & 0.005 & -0.033 & 0.577 & 0.003 & -0.009 & 0.310 & 0.264 \\
\hline$\tilde{S}_{2}$ & 0.027 & 0.707 & 0.005 & -0.033 & 0.577 & 0.003 & -0.009 & 0.309 & 0.263 \\
\hline$\tilde{S}_{3}$ & -0.055 & -0.001 & -0.011 & 0.066 & 0.578 & -0.005 & 0.017 & -0.618 & -0.525 \\
\hline
\end{tabular}

[16] M. Cvetič et al, ref. [5]; P. Langacker and J. Wang, Phys. Rev. D 58, 115010 (1998); G. Cleaver, M. Cvetič, J. R. Espinosa, L. L. Everett, P. Langacker and J. Wang, Phys. Rev. D 59, 115003 (1999).

[17] L. L. Everett, P. Langacker, M. Plümacher and J. Wang, Phys. Lett. B 477, 233 (2000).

[18] K. S. Babu, C. Kolda and J. March-Russell, Phys. Rev. D 57, 6788 (1998); K. R. Dienes, C. Kolda and J. March-Russell, Nucl. Phys. B 492, 104 (1997); F. del Aguila, G. D. Coughlan and M. Quiros, Nucl. Phys. B 307, 633 (1988) [Erratum-ibid. B 312, 751 (1988)]; B. Holdom, Phys. Lett. B 166, 196 (1986).

[19] U. Ellwanger and M. Lindner, Phys. Lett. B 301, 365 (1993); U. Ellwanger and C. Hugonie, hep-ph/0006222; hep-ph/9909260.

[20] See, for example, G. Abbiendi et al. [OPAL Collaboration], Eur. Phys. J. C 14, 187 (2000), Erratum-ibid. C 16, 707 (2000); A. Heister et al. [ALEPH Collaboration], hep-ex/0203020; M. Begalli [DELPHI Collaboration], Braz. J. Phys. 31, $223(2001)$.

[21] A. Bartl, H. Fraas, W. Majerotto and B. Mösslacher, Z. Phys. C 55, 257 (1992).

[22] B. de Carlos and J. R. Espinosa, Phys. Lett. B 407, 12 (1997).

[23] P. Langacker and M. Plümacher, Phys. Rev. D 62, 013006 (2000). 
Table 6: $\quad$ Same as Table 5, except $M_{i}>0$.

\begin{tabular}{|c|c|c|c|c|c|c|c|c|c|}
\hline Fields & $\tilde{\chi}_{1}^{0}$ & $\tilde{\chi}_{2}^{0}$ & $\tilde{\chi}_{3}^{0}$ & $\tilde{\chi}_{4}^{0}$ & $\tilde{\chi}_{5}^{0}$ & $\tilde{\chi}_{6}^{0}$ & $\tilde{\chi}_{7}^{0}$ & $\tilde{\chi}_{8}^{0}$ & $\tilde{\chi}_{9}^{0}$ \\
\hline$\tilde{B}^{\prime}$ & 0.0 & 0.0 & 0.0 & 0.0 & 0.001 & 0.002 & -0.004 & 0.659 & 0.752 \\
\hline$\tilde{B}$ & 0.036 & 0.290 & 0.001 & 0.0 & -0.001 & 0.955 & -0.048 & -0.001 & -0.001 \\
\hline$\tilde{W}_{3}^{0}$ & -0.028 & -0.217 & -0.001 & 0.0 & 0.001 & 0.116 & 0.969 & 0.002 & 0.002 \\
\hline$\tilde{H}_{1}^{0}$ & -0.279 & 0.697 & 0.002 & 0.0 & 0.608 & -0.192 & 0.170 & -0.029 & 0.026 \\
\hline$\tilde{H}_{2}^{0}$ & -0.440 & -0.605 & -0.002 & 0.0 & 0.606 & 0.192 & -0.172 & 0.059 & -0.054 \\
\hline$\tilde{S}$ & 0.849 & -0.111 & 0.0 & 0.0 & 0.513 & 0.002 & -0.001 & -0.046 & 0.039 \\
\hline$\tilde{S}_{1}$ & 0.032 & 0.031 & -0.707 & 0.577 & 0.003 & -0.009 & 0.008 & 0.306 & -0.268 \\
\hline$\tilde{S}_{2}$ & 0.031 & 0.026 & 0.707 & 0.577 & 0.003 & -0.009 & 0.008 & 0.305 & -0.268 \\
\hline$\tilde{S}_{3}$ & -0.062 & -0.057 & -0.001 & 0.578 & -0.005 & 0.018 & -0.016 & -0.610 & 0.535 \\
\hline
\end{tabular}

Table 7: Same as Table 5, except $h=0.75$.

\begin{tabular}{|c|c|c|c|c|c|c|c|c|c|}
\hline Fields & $\tilde{\chi}_{1}^{0}$ & $\tilde{\chi}_{2}^{0}$ & $\tilde{\chi}_{3}^{0}$ & $\tilde{\chi}_{4}^{0}$ & $\tilde{\chi}_{5}^{0}$ & $\tilde{\chi}_{6}^{0}$ & $\tilde{\chi}_{7}^{0}$ & $\tilde{\chi}_{8}^{0}$ & $\tilde{\chi}_{9}^{0}$ \\
\hline$\tilde{B}^{\prime}$ & 0.001 & 0.0 & 0.001 & -0.002 & 0.0 & -0.003 & -0.003 & 0.662 & 0.750 \\
\hline$\tilde{B}$ & -0.005 & 0.0 & 0.984 & -0.065 & 0.0 & -0.052 & -0.159 & -0.002 & -0.001 \\
\hline$\tilde{W}_{3}^{0}$ & 0.006 & 0.0 & 0.117 & 0.734 & 0.0 & 0.635 & 0.211 & 0.003 & 0.002 \\
\hline$\tilde{H}_{1}^{0}$ & -0.352 & 0.0 & 0.098 & 0.279 & 0.0 & -0.564 & 0.685 & -0.029 & 0.026 \\
\hline$\tilde{H}_{2}^{0}$ & -0.372 & 0.0 & -0.096 & 0.517 & 0.0 & -0.353 & -0.674 & 0.059 & -0.054 \\
\hline$\tilde{S}$ & 0.856 & 0.001 & 0.003 & 0.334 & 0.0 & -0.389 & -0.020 & -0.046 & 0.039 \\
\hline$\tilde{S}_{1}$ & 0.029 & -0.707 & 0.005 & -0.005 & 0.576 & -0.008 & 0.032 & 0.305 & -0.270 \\
\hline$\tilde{S}_{2}$ & 0.027 & 0.707 & 0.005 & -0.005 & 0.577 & -0.008 & 0.033 & 0.304 & -0.269 \\
\hline$\tilde{S}_{3}$ & -0.056 & -0.001 & -0.010 & 0.009 & 0.578 & 0.015 & -0.065 & -0.608 & 0.537 \\
\hline
\end{tabular}


Table 8: Same as Table 5, except $h=0.75$ and $M_{i}>0$.

\begin{tabular}{|c|c|c|c|c|c|c|c|c|c|}
\hline Fields & $\tilde{\chi}_{1}^{0}$ & $\tilde{\chi}_{2}^{0}$ & $\tilde{\chi}_{3}^{0}$ & $\tilde{\chi}_{4}^{0}$ & $\tilde{\chi}_{5}^{0}$ & $\tilde{\chi}_{6}^{0}$ & $\tilde{\chi}_{7}^{0}$ & $\tilde{\chi}_{8}^{0}$ & $\tilde{\chi}_{9}^{0}$ \\
\hline$\tilde{B}^{\prime}$ & 0.001 & 0.0 & 0.0 & 0.0 & 0.002 & 0.001 & -0.004 & 0.662 & 0.750 \\
\hline$\tilde{B}$ & 0.029 & -0.001 & 0.388 & 0.0 & 0.920 & -0.001 & -0.055 & -0.001 & -0.001 \\
\hline$\tilde{W}_{3}^{0}$ & -0.020 & 0.001 & -0.236 & 0.0 & 0.157 & 0.001 & 0.959 & 0.002 & 0.002 \\
\hline$\tilde{H}_{1}^{0}$ & -0.310 & -0.002 & 0.655 & 0.0 & -0.254 & 0.609 & 0.195 & -0.029 & 0.026 \\
\hline$\tilde{H}_{2}^{0}$ & -0.411 & 0.001 & -0.596 & 0.0 & 0.253 & 0.606 & -0.197 & 0.059 & -0.054 \\
\hline$\tilde{S}$ & 0.853 & 0.001 & -0.073 & 0.0 & 0.005 & 0.512 & -0.002 & -0.046 & 0.039 \\
\hline$\tilde{S}_{1}$ & 0.030 & -0.707 & 0.027 & 0.576 & -0.012 & 0.003 & 0.009 & 0.305 & -0.270 \\
\hline$\tilde{S}_{2}$ & 0.029 & 0.707 & 0.030 & 0.577 & -0.012 & 0.003 & 0.009 & 0.304 & -0.269 \\
\hline$\tilde{S}_{3}$ & -0.060 & -0.001 & -0.057 & 0.578 & 0.024 & -0.005 & -0.019 & -0.608 & 0.537 \\
\hline
\end{tabular}

Table 9: The eigenvectors for the CP even Higgses in Model I with $h=0.5$.

\begin{tabular}{|c|c|c|c|c|c|c|}
\hline Fields & $H_{1}^{0}$ & $H_{2}^{0}$ & $H_{3}^{0}$ & $H_{4}^{0}$ & $H_{5}^{0}$ & $H_{6}^{0}$ \\
\hline$H_{1}^{0 r}$ & 0.487 & 0.028 & 0.0 & 0.506 & 0.710 & 0.039 \\
\hline$H_{2}^{0 r}$ & 0.512 & 0.029 & 0.0 & 0.492 & -0.699 & -0.080 \\
\hline$S^{0 r}$ & -0.704 & 0.001 & 0.0 & 0.707 & -0.024 & 0.060 \\
\hline$S_{1}^{0 r}$ & -0.043 & 0.576 & -0.707 & -0.008 & 0.034 & -0.407 \\
\hline$S_{2}^{0 r}$ & -0.042 & 0.576 & 0.708 & -0.007 & 0.034 & -0.406 \\
\hline$S_{3}^{0 r}$ & 0.037 & 0.579 & 0.0 & -0.036 & -0.068 & 0.811 \\
\hline
\end{tabular}


Table 10: Same as Table 9, except $h=0.75$.

\begin{tabular}{|c|c|c|c|c|c|c|}
\hline Fields & $H_{1}^{0}$ & $H_{2}^{0}$ & $H_{3}^{0}$ & $H_{4}^{0}$ & $H_{5}^{0}$ & $H_{6}^{0}$ \\
\hline$H_{1}^{0 r}$ & 0.479 & 0.028 & -0.001 & 0.507 & -0.715 & 0.039 \\
\hline$H_{2}^{0 r}$ & 0.519 & 0.029 & 0.0 & 0.492 & 0.694 & -0.081 \\
\hline$S^{0 r}$ & -0.704 & 0.001 & 0.0 & 0.707 & 0.033 & 0.060 \\
\hline$S_{1}^{0 r}$ & -0.043 & 0.576 & -0.706 & -0.008 & -0.034 & -0.407 \\
\hline$S_{2}^{0 r}$ & -0.042 & 0.576 & 0.708 & -0.007 & -0.034 & -0.406 \\
\hline$S_{3}^{0 r}$ & 0.038 & 0.579 & -0.001 & -0.036 & 0.067 & 0.811 \\
\hline
\end{tabular}

Table 11: The eigenvectors for the CP odd Higgses in Model I with $h=0.5 . G_{1,2}^{0}$ are mixtures of the unphysical states absorbed by the $Z$ and $Z^{\prime}$.

\begin{tabular}{|c|c|c|c|c|c|c|}
\hline Fields & $G_{1}^{0}$ & $G_{2}^{0}$ & $A_{1}^{0}$ & $A_{2}^{0}$ & $A_{3}^{0}$ & $A_{4}^{0}$ \\
\hline$H_{1}^{0 i}$ & -0.665 & 0.212 & 0.0 & -0.322 & -0.013 & 0.639 \\
\hline$H_{2}^{0 i}$ & 0.670 & -0.256 & 0.0 & -0.314 & -0.013 & 0.623 \\
\hline$S^{0 i}$ & 0.020 & 0.057 & 0.0 & 0.891 & -0.001 & 0.451 \\
\hline$S_{1}^{0 i}$ & -0.135 & -0.386 & 0.707 & 0.023 & 0.576 & 0.010 \\
\hline$S_{2}^{0 i}$ & -0.135 & -0.384 & -0.707 & 0.023 & 0.578 & 0.010 \\
\hline$S_{3}^{0 i}$ & 0.269 & 0.768 & 0.001 & -0.059 & 0.578 & 0.008 \\
\hline
\end{tabular}


Table 12: Same as Table 11, except $h=0.75$.

\begin{tabular}{|c|c|c|c|c|c|c|}
\hline Fields & $G_{1}^{0}$ & $G_{2}^{0}$ & $A_{1}^{0}$ & $A_{2}^{0}$ & $A_{3}^{0}$ & $A_{4}^{0}$ \\
\hline$H_{1}^{0 i}$ & -0.696 & -0.013 & 0.0 & -0.322 & -0.013 & 0.641 \\
\hline$H_{2}^{0 i}$ & 0.718 & -0.026 & 0.0 & -0.312 & -0.013 & 0.622 \\
\hline$S^{0 i}$ & 0.001 & 0.060 & 0.0 & 0.891 & 0.0 & 0.449 \\
\hline$S_{1}^{0 i}$ & -0.004 & -0.409 & 0.708 & 0.023 & 0.576 & 0.010 \\
\hline$S_{2}^{0 i}$ & -0.004 & -0.407 & -0.707 & 0.023 & 0.578 & 0.010 \\
\hline$S_{3}^{0 i}$ & 0.008 & 0.814 & 0.002 & -0.059 & 0.578 & 0.008 \\
\hline
\end{tabular}

